\title{
Increased expression of (pro)renin receptor does not cause hypertension or cardiac and renal fibrosis in mice
}

\author{
Alva Rosendahl ${ }^{1}$, Gianina Niemann ${ }^{1}$, Sascha Lange ${ }^{1}$, Erfan Ahadzadeh ${ }^{1}$, Christian Krebs ${ }^{1}$, Aurelie Contrepas ${ }^{2,3,4,11}$, \\ Harry van Goor ${ }^{5}$, Thorsten Wiech ${ }^{6}$, Michael Bader ${ }^{7}$, Michael Schwake ${ }^{8}$, Judith Peters ${ }^{9}$, Rolf Stahl ${ }^{1}$, \\ Geneviève Nguyen ${ }^{2,3,4,10}$ and Ulrich O Wenzel ${ }^{1,10}$
}

Binding of renin and prorenin to the (pro)renin receptor (PRR) increases their enzymatic activity and upregulates the expression of pro-fibrotic genes in vitro. Expression of PRR is increased in the heart and kidney of hypertensive and diabetic animals, but its causative role in organ damage is still unclear. To determine whether increased expression of PRR is sufficient to induce cardiac or renal injury, we generated a mouse that constitutively overexpresses PRR by knocking-in the Atp6ap2/PRR gene in the hprt locus under the control of a CMV immediate early enhancer/chicken betaactin promoter. Mice were backcrossed in the C57BI/6 and FVB/N strain and studied at the age of 12 months. In spite of a 25- to 80-fold renal and up to 400-fold cardiac increase in Atp6ap2/PRR expression, we found no differences in systolic blood pressure or albuminuria between wild-type and PRR overexpressing littermates. Histological examination did not show any renal or cardiac fibrosis in mutant mice. This was supported by real-time PCR analysis of inflammatory markers as well as of pro-fibrotic genes in the kidney and collagen in cardiac tissue. To determine whether the concomitant increase of renin would trigger fibrosis, we treated PRR overexpressing mice with the angiotensin receptor- 1 blocker losartan over a period of 6 weeks. Renin expression increased eightfold in the kidney but no renal injury could be detected. In conclusion, our results suggest no major role for PRR in organ damage per se or related to its function as a receptor of renin.

Laboratory Investigation (2014) 94, 863-872; doi:10.1038/labinvest.2014.83; published online 21 July 2014

The binding of renin and prorenin to the (pro)renin receptor (PRR) increases the catalytic activity of renin and activates prorenin non-proteolytically by unfolding the pro-segment and unmasking the active site. Binding of renin and prorenin to PRR also induces the phosphorylation of mitogen-activated protein kinase $\mathrm{p} 42 / \mathrm{p} 44$ leading to upregulation of profibrotic genes in vitro. ${ }^{1,2}$ The discovery of PRR provided a long-sought function for prorenin and a potential role of renin and prorenin in organ damage independent of angiotensin (Ang) II. But unlike other components of the renin angiotensin system (RAS), total knockout of PRR gene, known as Atp6ap2, is impossible. The recent generation of Atp6ap2/PRR-floxed mice raised the hope that the cardiovascular and renal functions of PRR could finally be clarified by performing tissue-specific deletion of Atp6ap2 in established models of disease. Unexpectedly, constitutive Atp6ap2 knockout in cardiomyocytes provoked severe heart failure, and knockout in podocytes provoked massive nephrotic syndrome, both resulting in early postnatal mortality. Mutant mice had profound organ remodeling

\footnotetext{
${ }^{1}$ Division of Nephrology, University Hospital Hamburg-Eppendorf, Hamburg, Germany; ${ }^{2}$ INSERM, U1050, Paris, France; ${ }^{3}$ Collège de France, Center for Interdisciplinary Research in Biology, Paris, France; ${ }^{4}$ CNRS, UMR 7241, College de France, Paris, France; ${ }^{5}$ Department of Pathology, University Medical Centre Groningen, University of Groningen, Groningen, The Netherlands; ${ }^{6}$ Division of Nephropathology, University Hospital Hamburg-Eppendorf, Hamburg, Germany; ${ }^{7}$ Max-Delbrück Center for Molecular Medicine, Berlin, Germany; ${ }^{8}$ Faculty of Chemistry, Biochemistry III, University of Bielefeld, Bielefeld, Germany and ${ }^{9}$ Institute of Biochemistry, ChristianAlbrechts-University, Kiel, Germany

Correspondence: G Nguyen, MD, PhD, Centre for Interdisciplinary Research in Biology, UMR INSERM U1050/CNRS 7241, Collège de France, 11 place Marcelin Berthelot, Paris, Cedex 05 75231, France.

E-mail: genevieve.nguyen@college-de-france.fr or Professor U Wenzel, MD, Division of Nephrology, Universitätsklinikum Hamburg-Eppendorf, Martinistr. 52, Hamburg 20246, Germany.

E-mail: wenzel@uke.de

${ }^{11}$ Present address: HealthExperts SAS, 6 Cité de l'ameublement, Paris 75011, France.

${ }^{10}$ These authors contributed equally to this work.

Received 19 October 2013; revised 28 March 2014; accepted 6 May 2014
} 
attributed to impaired autophagy and deficient lysosomal acidification related to the function of PRR as an accessory subunit of the vacuolar $\mathrm{H}^{+}$-ATPase (V-ATPase) ${ }^{3-5}$ On the other hand, increased PRR was observed in ischemic nephropathy ${ }^{6,7}$ glomerulosclerosis, ${ }^{8}$ diabetic nephropathy, ${ }^{9}$ diabetic retinopathy, ${ }^{10}$ cardiac hypertrophy, ${ }^{11}$ and heart failure. ${ }^{12}$ In these models, the role of PRR was suggested, based on the use of a peptide that is supposed to block (pro)renin binding to PRR. But these results remain highly controversial. Therefore, to establish whether increased PRR could be responsible for organ damage, we generated transgenic mice with ubiquitous overexpression of mouse PRR. We used two different genetic backgrounds, C57Bl/6 and $\mathrm{FVB} / \mathrm{N}$, and we studied blood pressure, cardiac, and renal changes in function and in morphology in 1-year-old animals. Moreover, we studied the additional effect of an increased renin synthesis while blocking Ang II signaling by treating PRR overexpressing mice with the AT-1 antagonist losartan.

\section{MATERIALS AND METHODS}

All animal procedures were approved by Institutional Animal Care and Use Committees and were in accordance with the European Guidelines for the care and use of experimental animals.

\section{Generation of Atp6ap2/PRR Mice}

The Atp6ap2/PRR mouse line was generated using the 'Quick Knock-inTM' technology conducted by genOway, France. Briefly, mouse PRR cDNA was cloned into genOway's readyto-use Hprt-targeting vector under the control of the ubiquitous CAG (CMV immediate early enhancer/chicken beta-actin promoter fusion) promoter. A loxP flanked stop cassette was included between the promoter and the transgene to allow the expression to be dependent upon the action of Cre recombinase. The stop cassette was then removed by mating the Atp6ap2/PRR mice with a Cre deleter, which allows Cre-mediated excision of loxP flanked genes in all tissues including germ cells. ${ }^{13}$ Thereafter, mice were backcrossed for more than nine generations on the C57Bl/6 and $\mathrm{FVB} / \mathrm{N}$ background. As the hprt vector targets the transgene into the $\mathrm{X}$-chromosome, male mice carrying the transgene are hemizygous for Atp6ap2/PRR.

\section{Animals}

Male wild type (Atp6ap2/PRR ${ }^{\mathrm{wt} / \mathrm{y}}$ ) and hemizygous (Atp6ap2/ $\left.P R R^{\text {lox/y }}\right)$ as well as female wild type (Atp6ap2/PRR $\left.{ }^{\mathrm{wt} / \mathrm{wt}}\right)$, heterozygous, (Atp6ap2/PRR $R^{\text {lox/wt }}$ ) and homozygous $\left(\right.$ Atp6ap2/PRR ${ }^{\text {lox/ lox }}$ ) mice were examined. Wild-type and transgenic littermates were kept during 1 year in the same cage to avoid separation of the microbiota. When indicated, 10 -week-old mice were treated with losartan (Sandoz, Germany) $250 \mathrm{mg} / \mathrm{l}$ in drinking water for 6 weeks before killing.

\section{Functional Analyses}

Systolic blood pressure was measured in conscious mice using computerized tail cuff plethysmography (Process Control Blood Pressure 2900-series; TSE Systems, Germany) as described. ${ }^{14,15}$ Mice were trained to get used to this procedure in advance and initial measurements were not incorporated. For measurement of albuminuria, mice were placed into metabolic cages for a 6-hour urine collection. Urine albumin was measured using a commercially available mouse-specific ELISA (E90-134; Bethyl Laboratories, USA), urine creatinine by an autoanalyzer (Hitachi 717; Roche, Germany). Albuminuria was calculated as mg albumin per mg creatinine.

\section{Organ Removal}

Mice were anesthetized by isoflurane inhalation and killed, the heart and the kidneys were removed.

\section{Plasma Measurements}

At the time of killing, blood was drawn by intracardiac puncture and BUN was determined by an autoanalyzer (Hitachi 717). For the quantitative determination of aldosterone, a competitive chemiluminescence assay using directly coated magnetic microparticles on an automated LIAISON system, (DiaSorin, Germany) was used. Cross-reactivity against other steroid substances was below $0.02 \%$. Soluble PRR was measured by ELISA (JP27782, IBL International, Hamburg, Germany).

\section{Tissue Analyses \\ Histology}

The heart and kidney were fixed with $4 \%$ neutral buffered formaldehyde and embedded in paraffin. Cardiac tissue was stained with Masson-trichrome and fibrosis scored from 0 to 3 as described. ${ }^{16}$ Kidney sections were stained with PAS reagent and glomerular injury was evaluated using a semiquantitative score between 0 and $3 .{ }^{15}$ Renin was stained as described. ${ }^{15}$ Real-time PCR was performed using SYBR Green (Qiagen, Germany) using the following primers: 18s Fwd 5'-CACGGC CGGTACAGTGAAAC-3', 18s Rev 5'-AGAGGAGCGAGCGAC CAAA-3'; PAI-1 Fwd 5'-GGACACCCTCAGCATGTTCA-3', PAI-1 Rev 5'-TCTGATGAGTTCAGCATCCAAGAT-3'; Renin Fwd 5'-GCTCTGGAGTCCTTGCACCTT-3', Renin Rev 5'-CT TGAGCGGGATTCGTTCAA-3'; PRR Fwd 5'-TCATTCGACA CATCCCTTGTGPRR-3', PRR Rev 5'-AGGTTATAGGGACTTT GGGTGTTCT-3'; Cox-2 Fwd 5'-TAAGCGAGGACCTGGGTT CA-3' ${ }^{\prime}$, Cox-2 Rev 5'-TGTCCAGAGTTTCACCATAAATGTG-3'; Collagen I a2 Fwd 5'-CCCCGGGACTCCTGGACTT-3', Collagen I a2 Rev 5'-GCTCCGACACGCCCTCTCTC-3'; Collagen IV Fwd 5'-TACCTGCCACTACTTCGCTAAC-3', Collagen IV Rev 5'-CGGATGGTGTGCTCTGGAAG-3'; TGF-beta Fwd 5'-CCG CAACAACGCCATCTATGATG-3', TGF-beta Rev 5'-GGGGGT CAGCAGCCGGTTAC-3'; CCL2 Fwd 5'-GGCTCAGCCAGA TGCAGTTAA-3', CCL2 Rev 5'-CCTACTCATTGGGATCATC TTGCT-3'. 


\section{Western Blotting}

Renal lysate was prepared using precellys beads (Bertin Technologies) following the manufacturer's instructions. Lysates were solubilized in Laemmli loading buffer, separated by a SDS-PAGE and analyzed by western blotting. AntiATP6AP2 (HPA003156, Sigma) antibody was used, which detects the full length and the N-terminal fragment of the PRR. Anti-actin antibody (A2066, Sigma) served as a loading control. A peroxidase-conjugated secondary antibody (antirabbit-POD, Dianova) allowed the detection of PRR by a chemiluminescence (Amersham). Mouse neuroblastoma cells (N2a) were used as positive control.

\section{Statistical Analysis}

All data are expressed as mean \pm s.e.m. For the statistical analysis, Graph Pad Prism 5.1 was used. The KolmogorovSmirnov test was used to test for normal distribution of the values. In normally distributed data sets, the $t$-test was used to analyze the statistical significance between two groups and one-way ANOVA with post hoc analyses by Newman-Keuls Multiple Comparison Test was used to test the significance between three or more groups. If the data were not normally distributed, the Mann-Whitney test was used instead of the $t$ - test for comparing two samples and the Kruskal-Wallis test was used for three or more groups. When $N$ was too small to test for normal distribution, the Mann-Whitney test or Kruskal-Wallis test were performed. Differences were considered statistically significant at $P<0.05$. For power calculation, the R Project for Statistical Computing (http:// www.r-project.org) was used.

\section{RESULTS}

\section{Transgene Expression}

A Southern blot analysis resulting in a wild type $(7.9 \mathrm{~kb})$ and knock-in band $(10.7 \mathrm{~kb})$ revealed the proper targeting of the transgene and is shown in Figure 1a. Real-time PCR analysis showed a 24- to 43-fold increased renal expression (Figure 1b) and a 243- to 419-fold increase of Atp6ap2/PRR expression in the heart in the C57Bl/6 strain (Figure 1c). The

Table 11 Year basal data Atp6ap2/PRR C57BI/6 mice

\begin{tabular}{lcccc}
\hline & Survival & $\begin{array}{c}\text { Body } \\
\text { weight }(\mathrm{g})\end{array}$ & $\begin{array}{c}\text { Relative kidney } \\
\text { weight }(\mathrm{mg} / \mathrm{g})\end{array}$ & $\begin{array}{c}\text { Relative } \\
\text { heart weight } \\
(\mathrm{mg} / \mathrm{g})\end{array}$ \\
\hline Male wild type & $6 / 6$ & $34.6 \pm 2.1$ & $5.8 \pm 0.3$ & $3.7 \pm 0.2$ \\
Male hemizygous & $3 / 3(5 / 5)^{\mathrm{a}}$ & $29.0 \pm 1.7$ & $7.4 \pm 0.5$ & $4.2 \pm 0.4$ \\
Female wild type & $6 / 6$ & $25.2 \pm 2.0$ & $6.6 \pm 0.6$ & $4.6 \pm 0.5$ \\
Female heterozygous & $6 / 6$ & $26.0 \pm 1.4$ & $6.6 \pm 0.2$ & $4.4 \pm 0.2$ \\
Female homozygous & $5 / 6$ & $23.3 \pm 0.7$ & $6.3 \pm 0.2$ & $4.4 \pm 0.1$
\end{tabular}

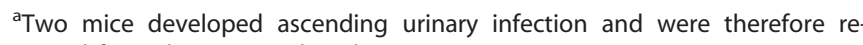
moved from the structural analysis.
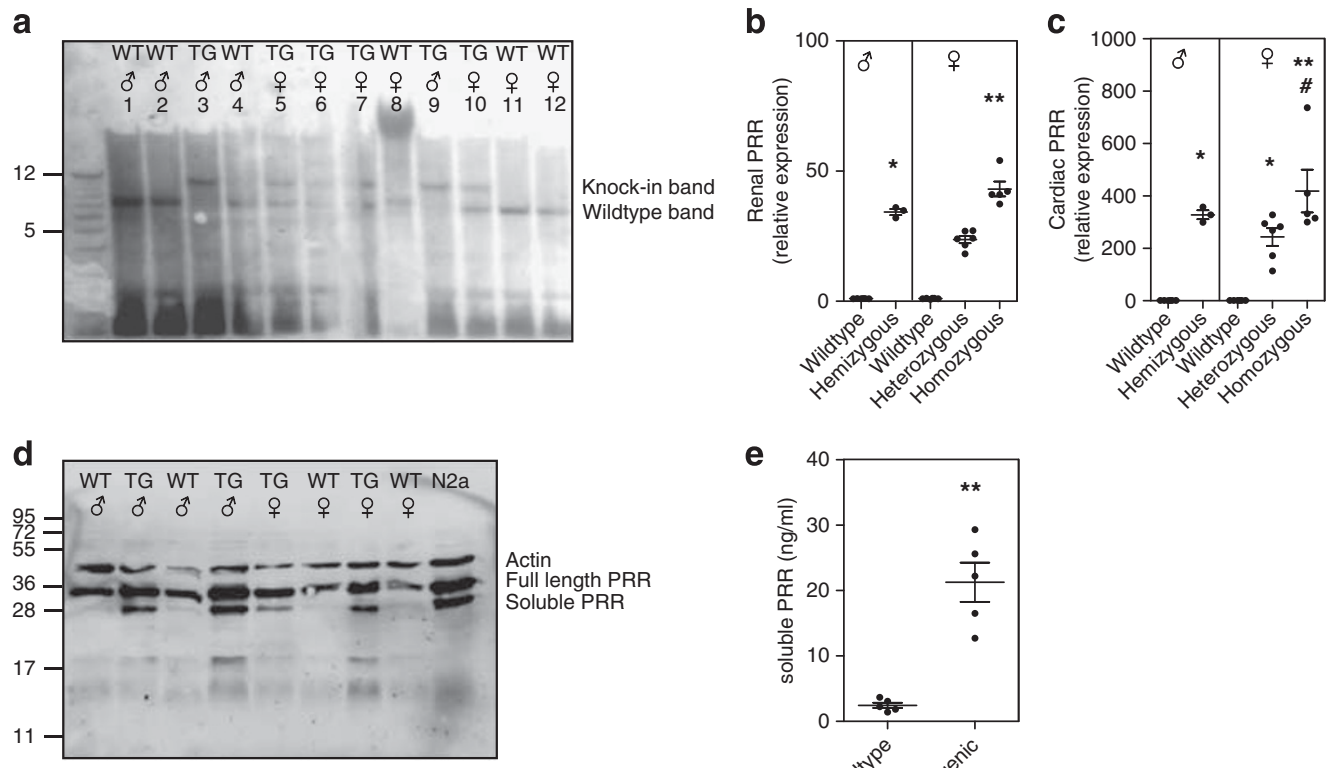

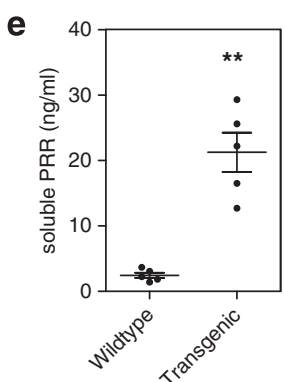

Figure 1 Characterization of PRR overexpression. (a) Southern blotting shows male wild-type and hemizygous mice with either the wild-type (mouse $1,2,4$ ) or the knock-in band (mouse 3,9). Female wild-type mice have the wild-type band (mouse 8,11,12) and female heterozygous mice exhibit both bands (mouse 5,6,7,10). (b, c) Real-time PCR shows renal and cardiac upregulation of the PRR of both genders. (d) Western blotting of renal lysate from wild-type and transgenic mice shows upregulation of the full length and soluble PRR in the kidney. (e) Plasma levels of soluble PRR were significantly increased in transgenic compared with wild-type mice. ${ }^{*} P<0.05$, ${ }^{* *} P<0.001$ vs wild type, ${ }^{\#} P<0.05$ vs heterozygous. 
a

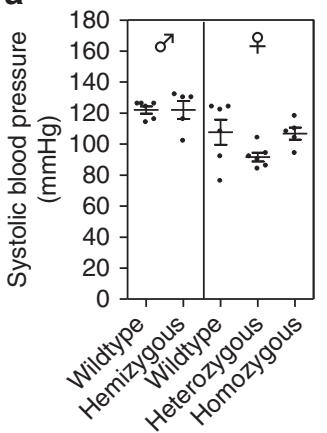

b

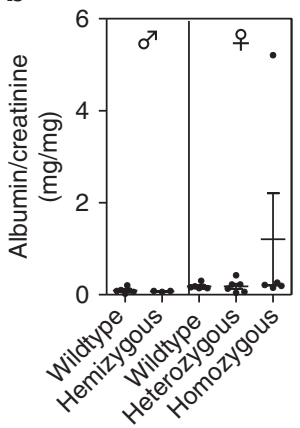

C
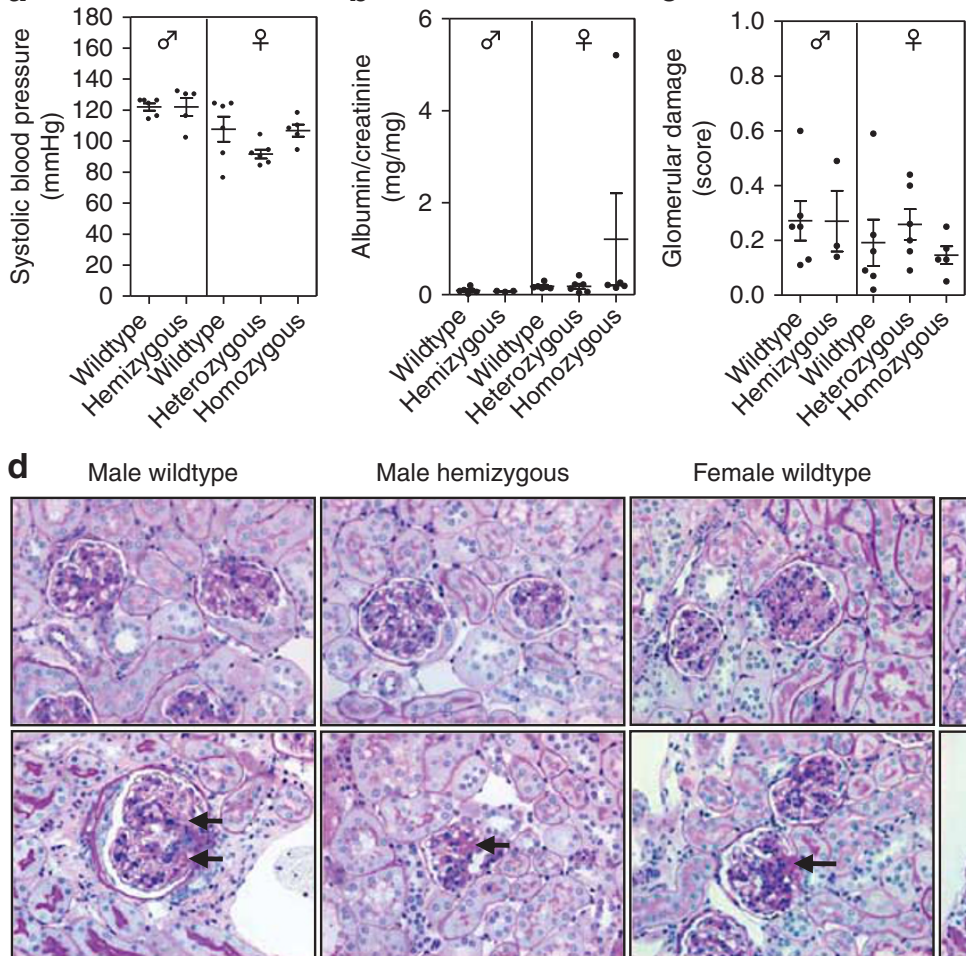

Male hemizygous

Female wildtype

Female heterozygous

Female homozygous
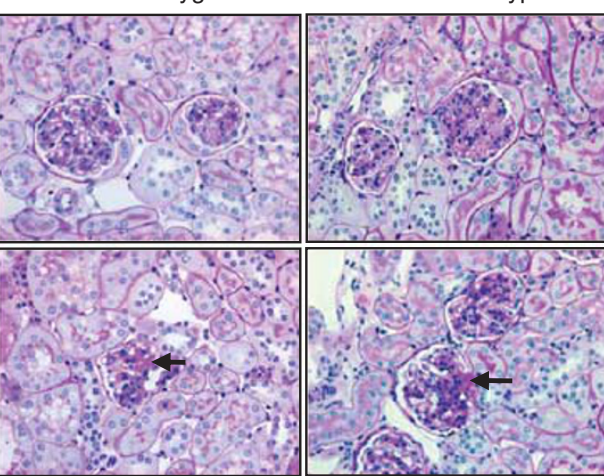

Fis pods?

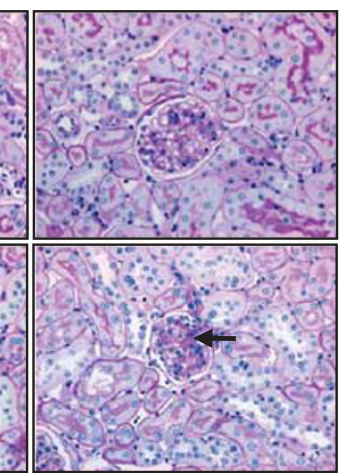

e Male wildtype
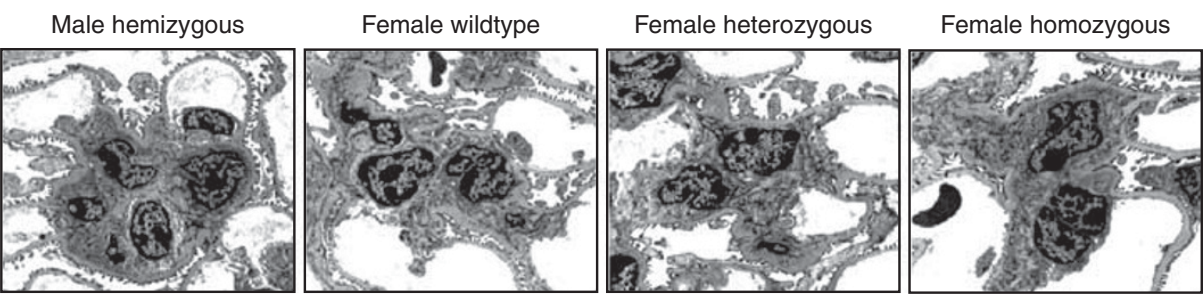

f
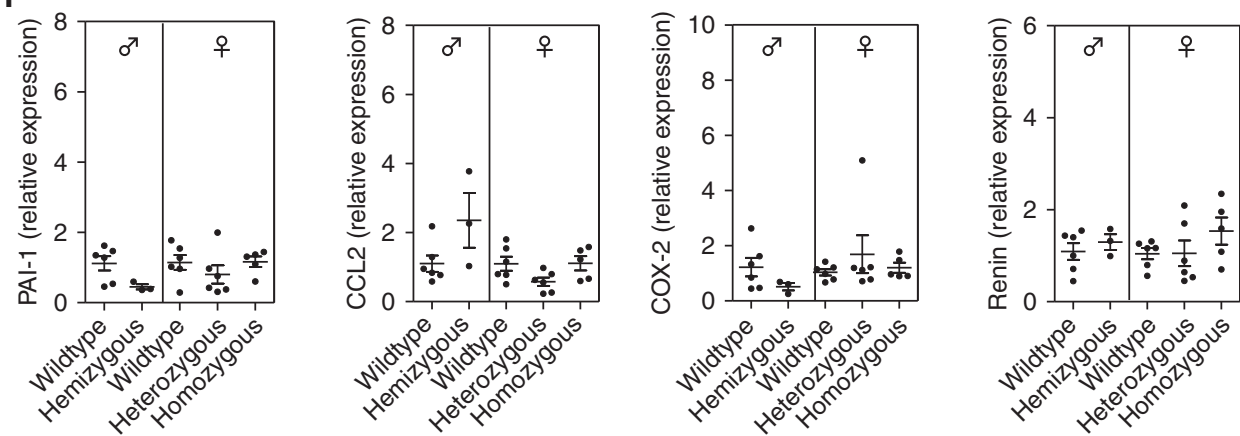

Figure 2 1-Year renal assessment of Atp6ap2/PRR C57BI/6 mice. (a) Systolic blood pressure values of one year old wild-type and transgenic mice of both genders. (b) No increased albuminuria was found in transgenic compared with wild-type mice. (c) Scoring of the glomerular changes revealed no difference between wild-type and transgenic mice. (d) Representative micrographs of PAS-stained sections (400-fold magnification). No glomerular or tubulointerstitial pathology was found. Occasionally mesangial widening or scarring was found in all five groups as shown in the micrographs below. (e) Electron microscopy revealed no ultrastructural evidence of podocyte or basal membrane damage. (f) Analysis of gene expression of PAI-1, CCL2, COX-2, and renin by real-time PCR revealed no significant difference between wild-type and transgenic mice.

same pattern was found in the $\mathrm{FVB} / \mathrm{N}$ strain (renal 27- to 79-fold, cardiac 235- to 478-fold overexpression). The increased expression was confirmed by western blotting of renal lysate using an antibody directed against the $\mathrm{N}$ termi- nus of PRR. Increased full length and soluble PRR expression could be detected in transgenic mice (densitometry corrected for actin: $2.8 \pm 0.1 v s 1.7 \pm 0.4, P<0.05$, Figure 1d). Moreover, compared with wild-type mice a ninefold increase of 

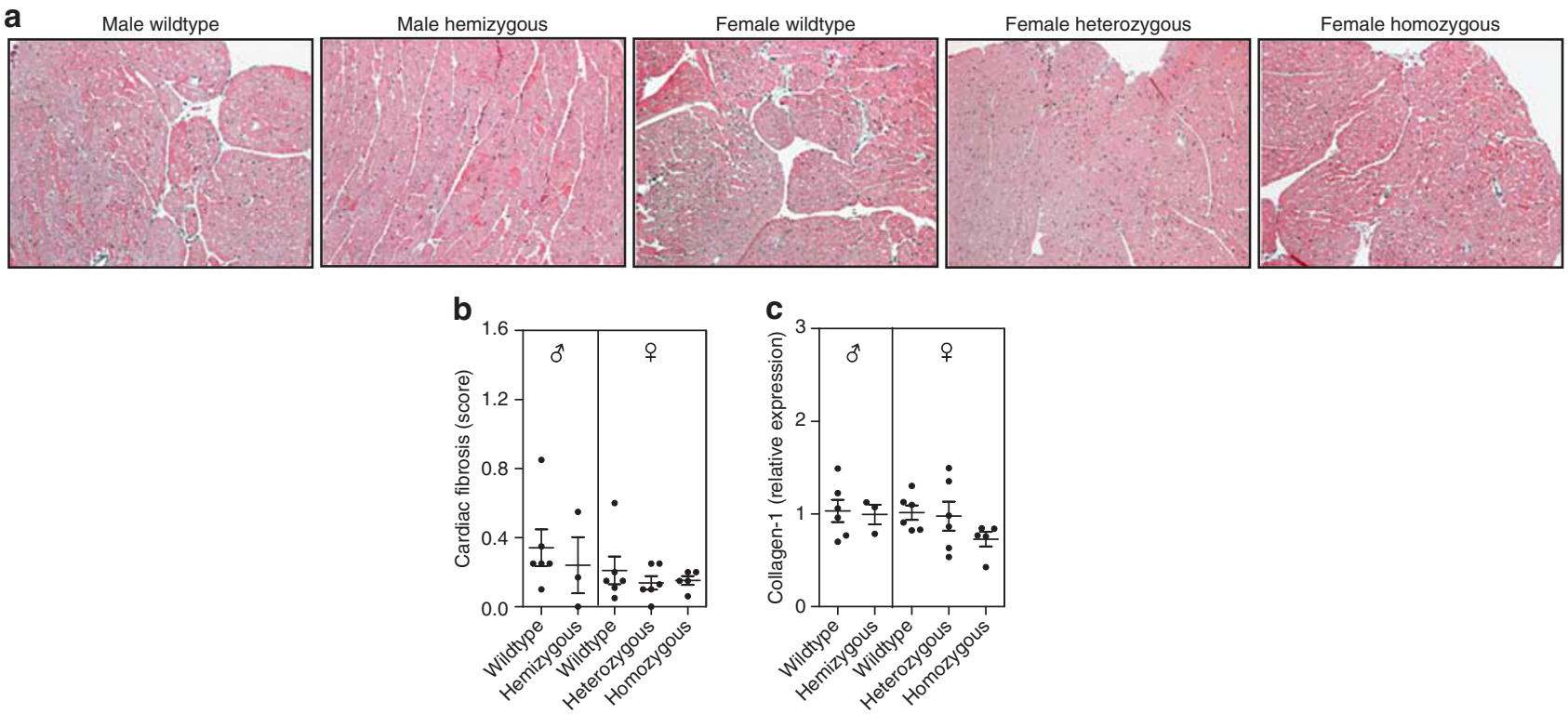

Figure 3 1-Year cardiac assessment of Atp6ap2/PRR C57BI/6 mice. (a) Masson-trichrome staining (200-fold magnification) shows intact myocytes without fibrosis in all five groups. (b) Semiquantitative scoring of cardiac fibrosis. (c) Analysis of collagen-1 expression by real-time PCR revealed no significant difference between wild-type and transgenic mice.

plasma soluble PRR levels was found in transgenic mice (Figure 1e).

\section{Assessment of Kidney Histology in Atp6ap2/PRR C57BI/6 Mice}

No significant differences in the body weight, heart and kidney weight (Table 1), or systolic blood pressure (Figure 2a) were observed regardless of gender and genotype. Albuminuria is a very sensitive marker of kidney dysfunction. At the age of 12 months, transgenic mice of both genders did not develop any increase of albuminuria compared with wild-type littermates (Figure 2b). Semiquantitative analysis of glomerulosclerosis showed no significant difference between wild-type and transgenic mice (Figure 2c). As shown on kidney sections stained by PAS, transgenic mice showed normal renal morphology (Figure 2d, upper panel). As expected in mice of 12 months of age, glomeruli with mild scarring or mesangial widening most often next to the vascular pole (arrows in Figure 2d, lower panel) were found occasionally in all genotypes. Electron microscopy showed no ultrastructural evidence of podocyte or basal membrane damage. Matrix expansion with mesangial deposition of electron dense material was equally observed in wild-type and in mutant mice (Figure 2e). Quantitative PCR analyses showed no significant differences between wild-type and mutant mice for PAI-1, CCL2, COX-2, and renin (Figure 2f). However, as there was a tendency that hemizygous mice express less PAI-1 than male wild-type mice, renal expression of TGF-beta and collagen IV was assessed in addition to PAI-1 expression, but no significant differences could be determined for these two parameters (TGF-beta: wild type $1.06 \pm 0.15$ versus hemizygous $1.19 \pm 0.66, P=0.9048$; collagen IV: wild type $1.02 \pm 0.09$ versus hemizygous $1.15 \pm 0.06, P=0.1667)$. Two male transgenic mice developed an ascending infection of the kidney with histological signs of pyelonephritis at the end of the year and were removed from the structural analysis. Owing to this fact the number of male mice analyzed was small. Thus, we confirmed that no renal injury was found in transgenic mice by analyzing another set of 12 male transgenic mice and 13 age-matched wild-type littermates. The data are shown in Supplementary Figure 1.

\section{Assessment of Cardiac Histology in Atp6ap2/PRR C57BI/6 Mice}

The heart is another important target of RAS-induced injury and an organ where RAS inhibition is beneficial. No exaggerated cardiac fibrosis was observed in transgenic mice compared with their respective wild-type (Figure 3a). This was supported by the scoring values (Figure $3 \mathrm{~b}$ ). The absence of cardiac injury was confirmed by measuring gene expression of collagen-1, which was not increased in transgenic mice (Figure 3c).

\section{Assessment of Atp6ap2/PRR in FVB/N Mice}

As mice of the FVB/N strain are more sensitive to develop renal disease in response to injury as recently shown by us, ${ }^{15,17}$ we also backcrossed the Atp6ap2/PRR mouse into the $\mathrm{FVB} / \mathrm{N}$ genetic background and examined the different genotypes of both genders at the age of 1 year. The results 
Table 21 Year basal data of Atp6ap2/PRR FVB/N mice

\begin{tabular}{|c|c|c|c|c|}
\hline & Survival & $\begin{array}{c}\text { Body } \\
\text { weight (g) }\end{array}$ & $\begin{array}{l}\text { Relative kid- } \\
\text { ney weight } \\
\quad(\mathrm{mg} / \mathrm{g})\end{array}$ & $\begin{array}{c}\text { Relative } \\
\text { heart weight } \\
\text { (mg/g) }\end{array}$ \\
\hline Male wild type & $4 / 5$ & $38.6 \pm 1.7$ & $6.6 \pm 0.5$ & $3.8 \pm 0.1$ \\
\hline Male hemizygous & $5 / 7$ & $36.5 \pm 1.7$ & $6.9 \pm 0.3$ & $3.9 \pm 0.2$ \\
\hline Female wild type & $5 / 8$ & $29.8 \pm 2.3$ & $5.7 \pm 0.3$ & $3.7 \pm 0.2$ \\
\hline Female heterozygous & $5 / 7$ & $30.2 \pm 1.5$ & $5.8 \pm 0.1$ & $4.3 \pm 0.2$ \\
\hline Female homozygous & $3 / 7$ & $27.7 \pm 2.0$ & $6.1 \pm 0.3$ & $3.5 \pm 0.3$ \\
\hline
\end{tabular}

are shown in Table 2 and Figure $4 \mathrm{a}-\mathrm{i}$ and are identical to the data in the C57Bl/6 strain. Neither male nor female transgenic mice developed arterial hypertension, altered renal function as assessed by plasma urea-N, glomerular or cardiac injury. In addition, no differences were found for plasma aldosterone levels that were measured, as increased aldosterone levels were reported in a PRR transgenic rat $^{18}$ (Figure 4i). A small but significant increase was found for albuminuria in heterozygous female mice compared with wild-type mice $(0.28 \pm 0.09$ versus $0.06 \pm 0.02 \mathrm{mg}$ albumin per $\mathrm{mg}$ creatinine, $P=0.0439$ ). No increase was seen in homozygous mice. As no other clue was found suggesting renal or cardiac injury in transgenic FVB mice, we believe that this finding was without biological relevance and by chance. As we have demonstrated previously that severe renal injury can be induced in $\mathrm{C} 57 \mathrm{Bl} / 6$ mice by administration of DOCA and Ang II and in FVB/N by $5 / 6$ nephrectomy (Supplementary Figure 2), ${ }^{15,16}$ the lack of any kind of renal injury in the PRR transgenic mice was not caused by the genetic background of the mice.

\section{Effect of Increased Renin by Treatment with Losartan}

To examine Ang-independent effects of renin and prorenin signaling through PRR, mice were treated for 6 weeks with the Ang receptor-1 antagonist losartan in order to stimulate renin expression. As expected, renin expression in the kidney was increased seven- to eightfold and four- to fivefold in $\mathrm{C} 57 \mathrm{Bl} / 6$ and $\mathrm{FVB} / \mathrm{N}$ mice, respectively (Figure 5a). The upregulation of renin expression was confirmed by renin immunohistochemistry. Figure $5 \mathrm{~b}$ shows low abundance of juxtaglomerular renin positivity in both genotypes and an increase after losartan treatment. In spite of this increase of renin, losartan-treated mice did not develop albuminuria (Figure 5c), glomerulosclerosis (Figure 5d), altered PAI-1 and CCL2 expression (Figure 5e), or change in renal function assessed by plasma urea-N (Figure 5f).

\section{DISCUSSION}

Renin and prorenin binding to PRR increases their enzymatic activity and triggers mitogen-activated protein kinase phos- phorylation. Both are established in vitro but not in vivo. ${ }^{1,2}$ These characteristics made PRR a potential therapeutic target to optimize the RAS blockade. Many attempts were made to establish the role of PRR in organ damage, alone or in association with increased renin or prorenin.

A particular attention was given to prorenin because it is increased in diabetic patients and its concentration correlates well with the presence and severity of microvascular complications. ${ }^{19}$ Experimental models with inducible or constitutive liver-specific expression of a prorenin transgene resulted in Ang II-dependent hypertension, but none of the transgenic models displayed microvascular damage as observed in diabetic patients. Also no cardiac or renal fibrosis was observed in spite of circulating prorenin levels up to 1000 times normal. ${ }^{20}$ This indicates that an increase of prorenin but normal PRR expression does not cause fibrosis. One could argue that this is due to a downregulation of PRR by prorenin itself ${ }^{21}$ but this hypothesis has never been confirmed in in vivo models with high levels of prorenin and/ or renin. Rather, the consensus is now that there is no evidence linking high prorenin to anything other than Ang II-dependent hypertension. ${ }^{22}$

The relevance of renin binding to PRR in vivo is being scrutinized. Given the nanomolar affinity of renin for PRR as opposed to the picomolar concentration of renin in plasma, it is currently uncertain whether such interaction truly occurs in vivo. The most likely site of interaction would be the juxtaglomerular apparatus, the site of renin synthesis, thus of highest renin concentration. Renin secretion is under tight control of Ang II levels through a negative feedback loop, and therefore RAS blockade causes a reactive rise in plasma renin concentration. ${ }^{15}$ Hence, RAS inhibition could in theory have adverse effects through activation of the PRR. In the present study, treatment with losartan for 6 weeks resulted in robust four- to eight-fold increase of renin expression in the cells of the juxtaglomerular apparatus concomitant to a 25- to 80-fold increase in PRR expression. Still, neither $\mathrm{C} 57 \mathrm{Bl} / 6$ nor the fibrosis susceptible $\mathrm{FVB} / \mathrm{N}$ strain showed any sign of renal injury.

Kaneshiro et al ${ }^{8,23}$ have generated a transgenic rat overexpressing human PRR and they reported an upregulation of cyclooxygenase 2, glomerulosclerosis, and proteinuria at the age of 28 weeks in the absence of hypertension or changes in renal RAS activity. Administration of Ang-converting enzyme inhibitor did not improve the glomerular damage. It was concluded that binding of rat prorenin to human PRR induces ERK1/2 activation, although it failed to get activated and that activation of PRR can induce organ damage independently of Ang II. Burckle et al ${ }^{18}$ overexpressed the human PRR in vascular smooth muscle cells in the rat and found increased blood pressure as well as increased plasma levels of aldosterone. Our results are in contradiction to those of Kaneshiro et al $l^{8,23}$ and the reasons for this discrepancy are not entirely clear. In the present study, we were very careful to analyze two different strains, $\mathrm{C} 57 \mathrm{Bl} / 6$ and the more 

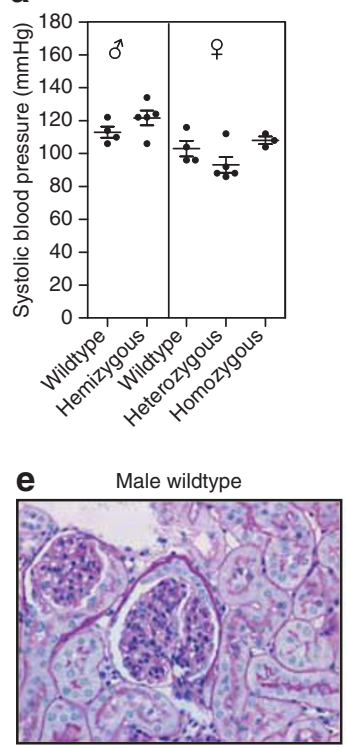

f

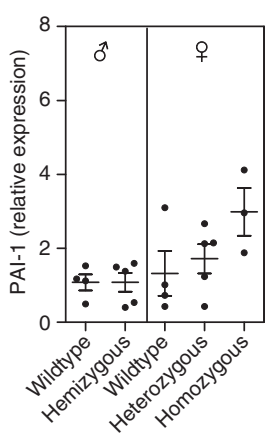

g

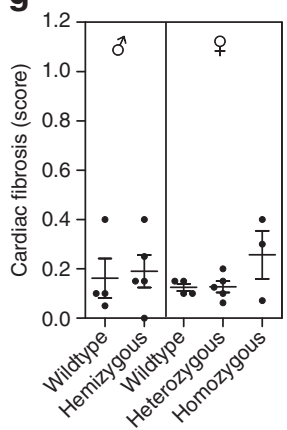

b

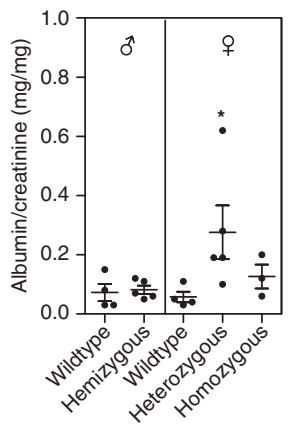

Male hemizygous

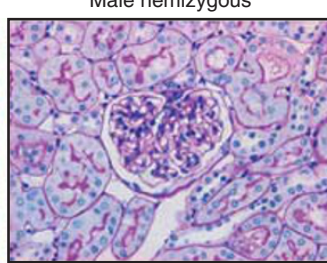

c

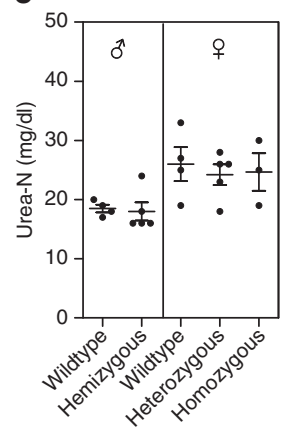

Female wildtype

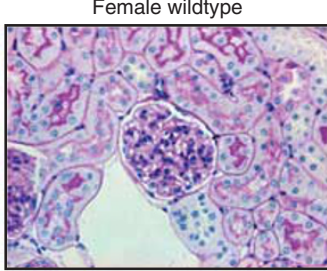

Female heterozygous
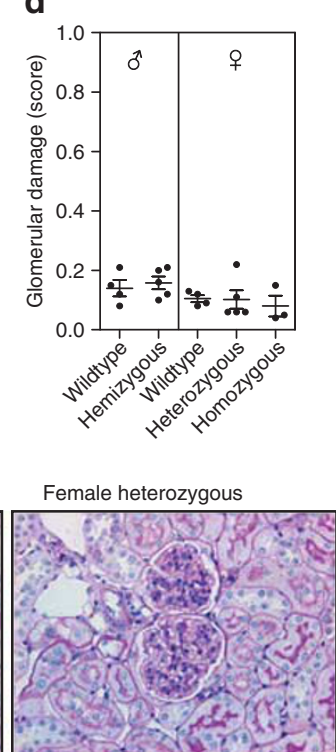

.
Female homozygous

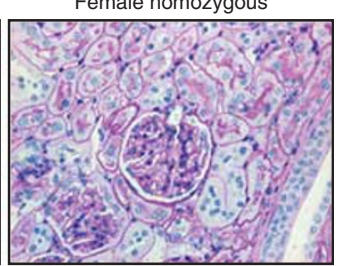

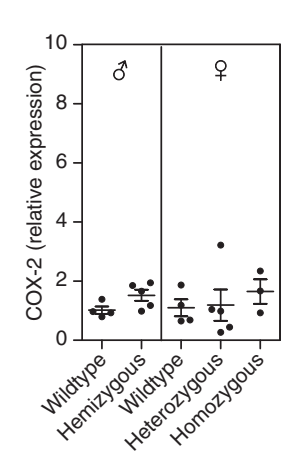

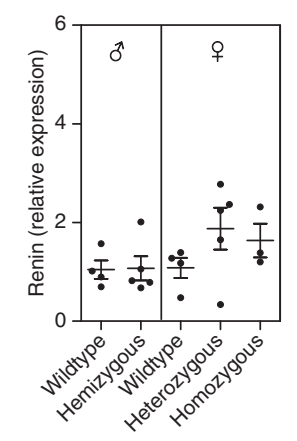

i
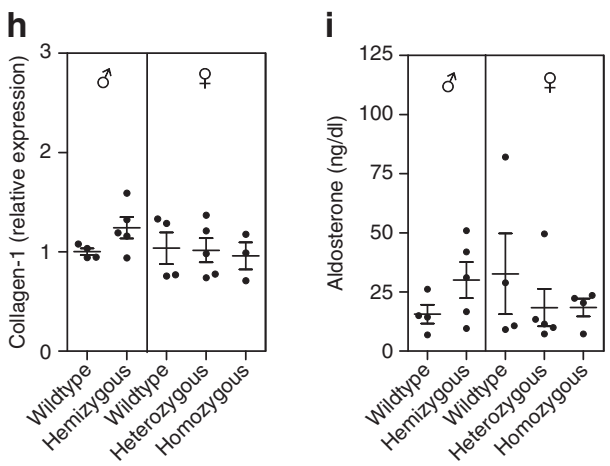

Figure 4 Renal and cardiac assessment of Atp6ap2/PRR FVB/N mice. (a) No significant differences were found for systolic blood pressure, (c) urea-N, and (d) glomerular damage between wild-type and transgenic littermates. (e) Representative micrographs of PAS-stained sections. (f) Real-time -PCR analysis of renal expression of PAI-1, CCL2, COX-2, and renin, (g) cardiac fibrosis, (h) expression of collagen-1 and (i) plasma aldosterone. A small but significant $(P=0.0439)$ increase of albuminuria was found in female heterozygous mice compared with wild-type mice (b).

susceptible to kidney damage strain FVB/N. Moreover, we overexpressed mouse PRR in mice, which is important, as species differences exist within the human, rat, and mouse RAS in terms of interaction of renin and prorenin with PRR. ${ }^{23,24}$ In addition, we compared wild-type and transgenic littermates to avoid differences other than those due to the overexpression of the PRR. Both gender were examined. Great care was taken not to overlook a renal phenotype. We used a number of quantitative and semiquantitative measurements to gauge the extent of renal injury in the present study. Renal injury was assessed by scoring of glomerular changes in PAS-stained renal sections, and the 


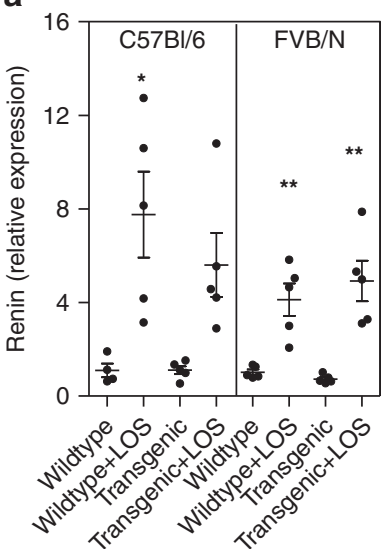

b
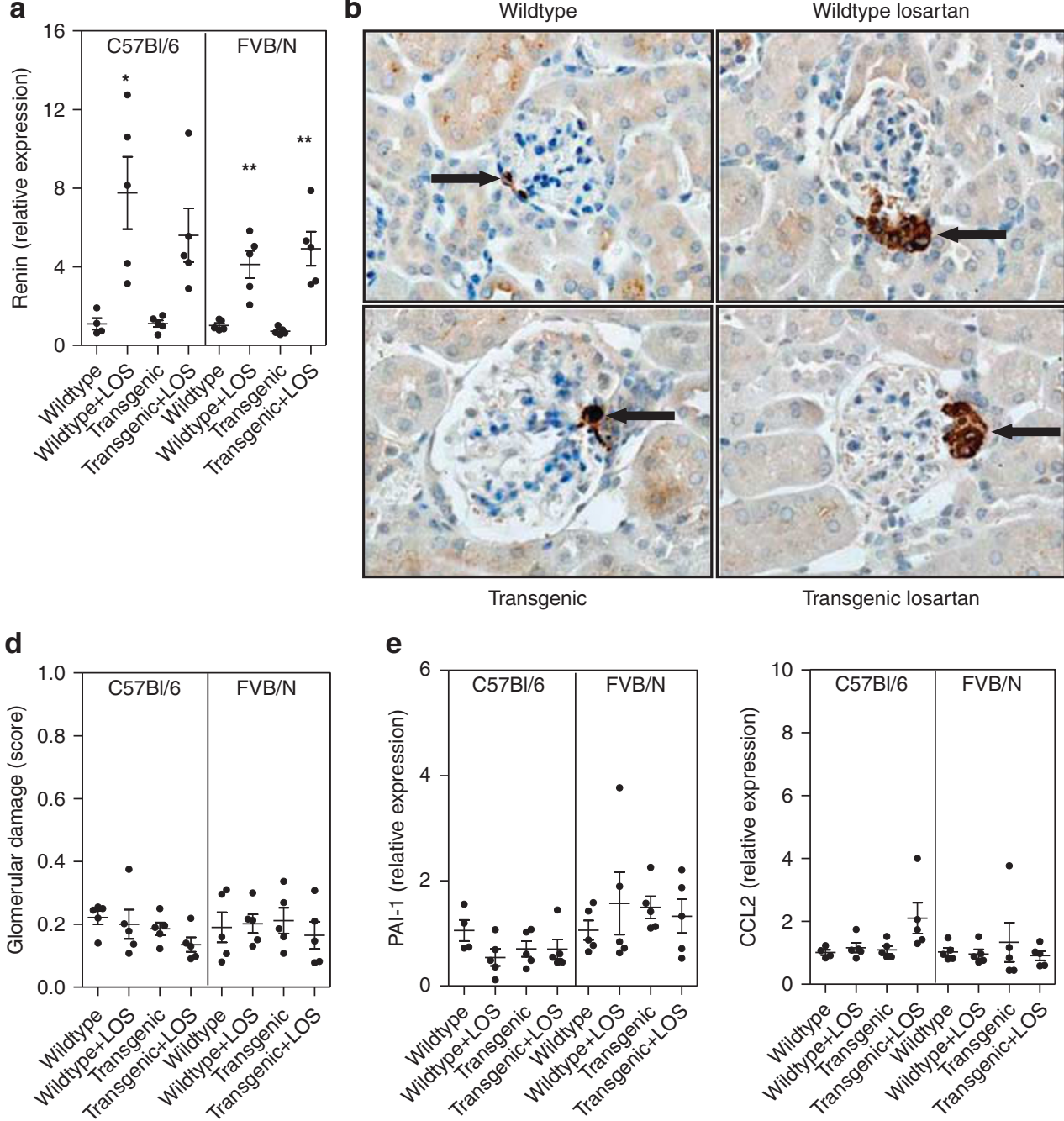

e

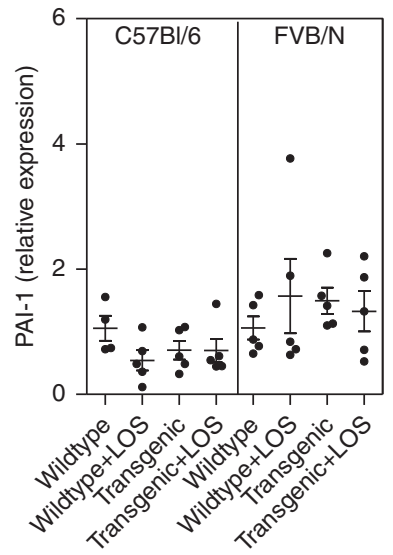

C
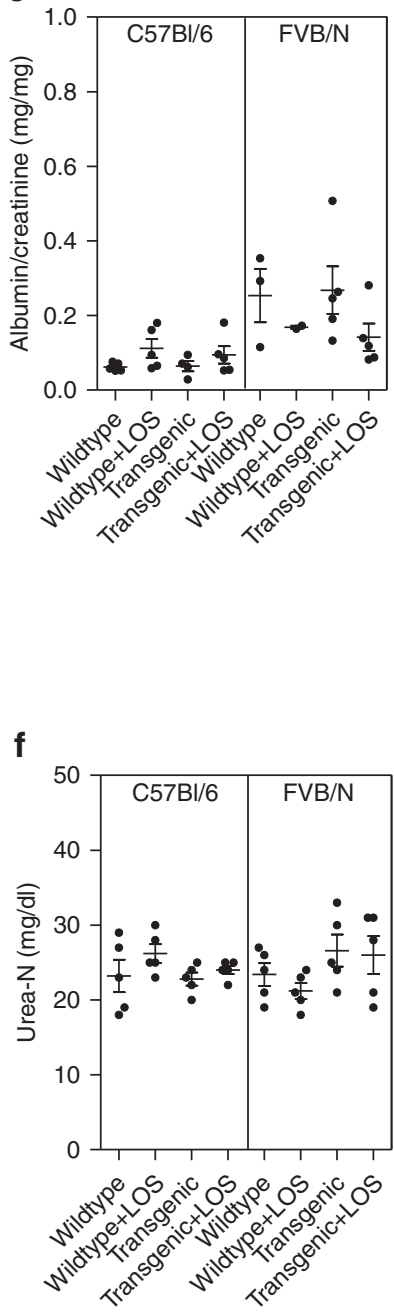

Figure 5 Effect of increased renin by treatment with losartan. (a) Increased renin expression measured by real-time PCR in the kidney of wild-type and transgenic mice after 6 weeks losartan in C57BI/6 and FVB/N mice. (b) Upregulation of renin expression was confirmed by renin immunohistochemistry. No increase in (c) albuminuria or (d) glomerular damage were found after treatment with losartan. (e) Also renal gene expression measured by realtime PCR of PAl-1 and CCL2 shows no induction of fibrosis or inflammation by losartan. (f) No changes in plasma urea-N were found. * $P<0.01$, $* * P<0.001$ vs untreated.

absence of injury in the transgenic mice was confirmed by markers of inflammation and fibrosis such as CCL2 and PAI-1. Also electron microscopy revealed no ultrastructural evidence of podocyte or glomerular basal membrane damage. Is it possible that the overexpression of the PRR was too mild in our model? This is unlikely as we report a 25- to 400-fold overexpression of PRR by real-time PCR whereas Kaneshiro et $a l^{8}$ reported by semiquantitative RT-PCR that one transgenic line has a 10-fold higher mRNA expression than another transgenic line, but no comparison with wild-type rats as well as no protein data were provided. In our PRR overexpressing mouse, plasma levels of soluble PRR are increased ninefold compared with wild-type mice. Increased plasma soluble PRR levels are correlated with renal dysfunction in patients with heart failure and predict gestational diabetes mellitus during the first trimester PRR. ${ }^{25,26}$ In contrast to the work by Burckle et al, ${ }^{18}$ plasma aldosterone levels were not increased in our transgenic mice. The strength of the present study is that we examined two strains of mice with different genetic backgrounds, analyses of the results in both genders and in three genotypes (hemi-, hetero- and homozygous). Owing to the high number of the different groups, the number of mice used in the different groups was small. Nevertheless, the present study shows no evidence of a biological relevant difference between wild-type and transgenic mice. However, the number of mice examined in each group might has been a limitation. Absence of evidence is not evidence of absence. We cannot completely exclude that the study was underpowered and that an increase in sample size would detect a meaningful effect of 
PRR overexpression. In Supplementary Figure 1 we compared 12 transgenic mice with 13 wild-type mice and found no difference. A post hoc power calculation with the observed data indicates that a sample size of 1100 mice in each group would give a $80 \%$ power to detect a significant difference for albuminuria and a sample size of 336 mice in each group for glomerular damage.

The stop-flox approach of the PRR transgene chosen in the present study allows cell- or organ-specific overexpression of PRR. However, as overexpression in all tissues did not result in a phenotype, it is unlikely that cell-specific overexpression for example in podocytes or cardiomyocytes would result in a phenotype.

Although the binding of renin and of prorenin to PRR is now undisputed, the absence of an effect of PRR overexpression indicates that the triad renin-prorenin-PRR should not be considered as a primary initiator of cardiovascular and renal damage. Rather, it might have an aggravating role in tissue damage due to inflammation or diabetes. The absence of an overt phenotype of Atp6ap2/PRR overexpression in our mice contrasts with the severe phenotype of mice with a conditional ablation of Atp6ap2/PRR in cardiomyocytes and podocytes that leads to a severe heart failure or massive nephrotic syndrome and death within 3-4 weeks. This was attributed to the deficient function of PRR as co-factor of the V-ATPase and leading to impaired lysosomal acidification and autophagy. Furthermore, PRR was found to be necessary for Wnt/beta-catenin and Wnt/planar cell polarity complex endocytosis and activation in acidic endosomes. ${ }^{27}$ Retrospectively, this explains why total knockout of Atp6ap2/PRR is embryonic lethal. Because of these multiple interacting proteins, renin, prorenin, V-ATPase, and proteins of the Wnt/beta-catenin and Wnt/planar cell polarity receptor complexes, ${ }^{28}$ PRR-deficient phenotypes are difficult to interpret. Compounds or knockout approaches that block specifically each interaction are needed to decipher the functions of PRR. In addition, it remains to be examined whether overexpression of PRR in combination with another hit-like high salt, diabetes, or chronic kidney disease will induce injury. Taken together, our results indicate that increased PRR alone or associated with increased renin does not cause organ damage independently of Ang II.

Supplementary Information accompanies the paper on the Laboratory Investigation website (http://www.laboratoryinvestigation.org)

\section{ACKNOWLEDGMENTS}

We thank M Reszka and S Gatzemeier for excellent technical assistance. In addition, we thank I Hermans-Borgmeyer for her valuable advice with transgenic mice and S Appelbaum for statistical advice.

\section{DISCLOSURE/CONFLICT OF INTEREST}

The authors declare no conflict of interest.

1. Huang $\mathrm{Y}$, Wongamorntham $\mathrm{S}$, Kasting J, et al. Renin increases mesangial cell transforming growth factor-beta1 and matrix proteins through receptor-mediated, angiotensin II-independent mechanisms. Kidney Int 2006;69:105-113.

2. Nguyen G, Delarue F, Burckle C, et al. Pivotal role of the renin/prorenin receptor in angiotensin II production and cellular responses to renin. J Clin Invest 2002;109:1417-1427.

3. Kinouchi K, Ichihara A, Sano M, et al. The (pro)renin receptor/ATP6AP2 is essential for vacuolar $\mathrm{H}+$-ATPase assembly in murine cardiomyocytes. Circ Res 2010;107:30-34.

4. Riediger F, Quack I, Qadri F, et al. Prorenin receptor is essential for podocyte autophagy and survival. J Am Soc Nephrol 2011;22: 2193-2202.

5. Oshima $Y$, Kinouchi K, Ichihara A, et al. Prorenin receptor is essential for normal podocyte structure and function. J Am Soc Nephrol 2011:22:2203-2212.

6. Krebs C, Hamming I, Sadaghiani S, et al. Antihypertensive therapy upregulates renin and (pro)renin receptor in the clipped kidney of Goldblatt hypertensive rats. Kidney Int 2007;72:725-730.

7. Krebs $C$, Weber $M$, Steinmetz $O$, et al. Effect of (pro)renin receptor inhibition by a decoy peptide on renal damage in the clipped kidney of Goldblatt rats. Kidney Int 2008;74:823-824.

8. Kaneshiro $\mathrm{Y}$, Ichihara A, Sakoda $\mathrm{M}$, et al. Slowly progressive, angiotensin II-independent glomerulosclerosis in human (pro)renin receptor-transgenic rats. J Am Soc Nephrol 2007;18:1789-1795.

9. Ichihara A, Suzuki F, Nakagawa T, et al. Prorenin receptor blockade inhibits development of glomerulosclerosis in diabetic angiotensin II type 1a receptor-deficient mice. J Am Soc Nephrol 2006;17:1950-1961.

10. Satofuka S, Ichihara A, Nagai N, et al. (Pro)renin receptor-mediated signal transduction and tissue renin-angiotensin system contribute to diabetes-induced retinal inflammation. Diabetes 2009;58:1625-1633.

11. Ichihara A, Kaneshiro Y, Takemitsu T, et al. Nonproteolytic activation of prorenin contributes to development of cardiac fibrosis in genetic hypertension. Hypertension 2006;47:894-900.

12. Moilanen AM, Rysa J, Serpi R, et al. (Pro)renin receptor triggers distinct angiotensin II-independent extracellular matrix remodeling and deterioration of cardiac function. PloS One 2012;7:e41404.

13. Schwenk F, Baron U, Rajewsky K. A cre-transgenic mouse strain for the ubiquitous deletion of loxP-flanked gene segments including deletion in germ cells. Nucleic Acids Res 1995;23:5080-5081.

14. Kirchhoff F, Krebs C, Abdulhag UN, et al. Rapid development of severe end-organ damage in C57BL/6 mice by combining DOCA salt and angiotensin II. Kidney Int 2008;73:643-650.

15. Fraune $C$, Lange $S$, Krebs $C$, et al. AT1 antagonism and renin inhibition in mice: pivotal role of targeting angiotensin II in chronic kidney disease. Am J Physiol Renal Physiol 2012;303:F1037-F1048.

16. Krebs C, Fraune C, Schmidt-Haupt R, et al. CCR5 deficiency does not reduce hypertensive end-organ damage in mice. Am J Hypertens 2012;25:479-486.

17. Benndorf RA, Krebs C, Hirsch-Hoffmann B, et al. Angiotensin II type 2 receptor deficiency aggravates renal injury and reduces survival in chronic kidney disease in mice. Kidney Int 2009;75: 1039-1049.

18. Burckle CA, Jan Danser AH, Muller DN, et al. Elevated blood pressure and heart rate in human renin receptor transgenic rats. Hypertension 2006;47:552-556.

19. Luetscher JA, Kraemer FB, Wilson DM, et al. Increased plasma inactive renin in diabetes mellitus. A marker of microvascular complications. $\mathrm{N}$ Engl J Med 1985;312:1412-1417.

20. Mercure C, Prescott G, Lacombe MJ, et al. Chronic increases in circulating prorenin are not associated with renal or cardiac pathologies. Hypertension 2009;53:1062-1069.

21. Schefe JH, Menk M, Reinemund J, et al. A novel signal transduction cascade involving direct physical interaction of the renin/prorenin receptor with the transcription factor promyelocytic zinc finger protein. Circ Res 2006;99:1355-1366.

22. Reudelhuber TL. The interaction between prorenin, renin and the (pro)renin receptor: time to rethink the role in hypertension. Curr Opin Nephrol Hypertens 2012;21:137-141.

23. Kaneshiro $Y$, Ichihara A, Takemitsu $T$, et al. Increased expression of cyclooxygenase-2 in the renal cortex of human prorenin receptor gene-transgenic rats. Kidney Int 2006;70:641-646.

24. Ganten $\mathrm{D}$, Wagner J, Zeh $\mathrm{K}$, et al. Species specificity of renin kinetics in transgenic rats harboring the human renin and angiotensinogen genes. Proc Natl Acad Sci USA 1992;89:7806-7810. 
25. Fukushima A, Kinugawa S, Homma T, et al. Increased plasma soluble (pro)renin receptor levels are correlated with renal dysfunction in patients with heart failure. Int J Cardiol 2013;168:4313-4314.

26. Watanabe N, Morimoto S, Fujiwara T, et al. Prediction of gestational diabetes mellitus by soluble (pro)renin receptor during the first trimester. J Clin Endocrinol Metab 2013;98:2528-2535.
27. Cruciat CM, Ohkawara B, Acebron SP, et al. Requirement of prorenin receptor and vacuolar $\mathrm{H}+$-ATPase-mediated acidification for Wnt signaling. Science 2010;327:459-463.

28. Hermle T, Saltukoglu D, Grunewald J, et al. Regulation of Frizzleddependent planar polarity signaling by a V-ATPase subunit. Curr Biol 2010;20:1269-1276. 